\title{
ESCALAS DE QUALIDADE DE VIDA APLICADAS AOS FAMILIARES DE PACIENTES ONCOLÓGICOS EM CUIDADOS PALIATIVOS: UMA REVISÃO INTEGRATIVA
}

\section{REVISÃO INTEGRATIVA}

COSTA, Susana Rocha ${ }^{1}$

BONAMIGO, Andréa Wander ${ }^{2}$

SILVEIRA, Luiza Maria de Oliveira Braga ${ }^{3}$

COSTA, Susana Rocha. BONAMIGO, Andréa Wander. SILVEIRA, Luiza Maria de Oliveira Braga. Escalas de qualidade de vida aplicadas aos familiares de pacientes oncológicos em cuidados paliativos: Uma revisão integrativa. Revista Científica Multidisciplinar Núcleo do Conhecimento. Ano 05, Ed. 11, Vol. 05, pp. 5365. Novembro de 2020. ISSN: 2448-0959, Link de acesso: https://www.nucleodoconhecimento.com.br/saude/pacientes-oncologicos

\section{RESUMO}

Pacientes oncológicos terminais recebem cuidados paliativos com o objetivo de gerenciar a dor e os sintomas, que dão um suporte ao paciente e aos seus familiares, que lidam diariamente com as emoções e o estresse da morte iminente. Objetivo: identificar e analisar os instrumentos dos estudos realizados no Brasil sobre qualidade

${ }^{1}$ Mestrado profissional em Ensino na Saúde. Especialização em andamento em Docência no Ensino Superior. Especialização em Saúde da Família. Graduação em Enfermagem.

2 Doutorado em Saúde Pública. Mestrado em Distúrbios da Comunicação. Especialização em Saúde Pública. Graduação em Fonoaudiologia. Graduação em Letras Português Inglês.

${ }^{3}$ Doutorado em Psicologia. Mestrado em Psicologia. Graduação em Psicologia. 
de vida dos familiares de pacientes oncológicos em Cuidados Paliativos. Metodologia: revisão integrativa da literatura. Resultados: foram selecionados 12 estudos que atenderam aos critérios de inclusão e exclusão. Os estudos foram na sua maioria qualitativos. No Brasil, a ferramenta mais utilizada para mensurar a qualidade de vida dos familiares/cuidadores de pacientes em cuidados paliativos são as entrevistas semiestruturadas que podem ser acompanhadas ou não de outros instrumentos de análise, como por exemplo: o grupo focal e o inventário de estresse. Considerações finais: Os familiares/cuidadores de pacientes oncológicos em fase terminal, que recebem cuidados paliativos, têm sua qualidade de vida comprometida devido ao estresse físico, psicológico e econômico, aos quais são submetidos nesse período.

Palavras-chave: Cuidados paliativos, câncer, qualidade de vida, cuidador, família.

\section{INTRODUÇÃO}

O esgotamento de terapêutica curativa levou a necessidade de desenvolver mecanismos que trouxessem qualidade de vida aos pacientes submetidos ao tratamento oncológico em fase terminal, sendo esse processo chamado de "Cuidados Paliativos". É nessa esfera de cuidados que são realizadas abordagens com vistas a aguardar o final da vida, com apoio psicológico, social, físico e espiritual (CARVALHO; PERSONS, 2012). Os cuidados paliativos são focalizados na qualidade de vida do paciente terminal e não no prolongamento da vida, aceitando a morte como sua fase final (RENALHO, 2017).

Os pacientes terminais e suas famílias encontram-se em um momento de sensibilidade, mas necessitam de suporte familiar. O apoio familiar será de extrema importância para que seja possível planejar os cuidados ao paciente e ter sucesso na realização do tratamento (ARAUJO et al., 2009).

A abordagem dos cuidados paliativos inicia-se com a avaliação abrangente de todos os domínios da vida do paciente. A comunicação e a tomada de decisões sobre questões difíceis devem incluir respostas emocionais dos familiares, planejando com antecedência as necessidades de comunicação de más notícias e os desafios destas. 
O planejamento antecipado de cuidados paliativos inclui a abordagem de valores, objetivos e preferências para a assistência ao paciente terminal, com o envolvimento familiar (DY; ISENBERG, 2017).

A família é a base estrutural para que esse cuidado seja efetivo. Esse grupo de pessoas que se formam por laços sanguíneos ou afetivos, são aqueles que estarão junto ao paciente terminal em distintos momentos do seu processo de adoecimento. Muitas vezes acompanham o paciente desde a descoberta da doença, durante todo o tratamento e até o final, quando se esgotaram as possibilidades de cura (ARAUJO et al., 2009; IDO; TADAKA, 2017).

Os cuidadores familiares são uma presença indispensável para os pacientes com câncer terminal. Suas tarefas de cuidados incluem apoio emocional, suporte às atividades da vida diária (AVDs), administração de medicamentos, provisão de nutrição e assistência com outros aspectos físicos do cuidado (CARVALHO; PERSON, 2012; ARAÚJO et al., 2009). No entanto, as responsabilidades de cuidar geram várias questões, incluindo conflitos entre seus papéis pessoais e sociais, restrições de suas atividades, tensão nas relações matrimoniais e familiares, sofrimento psicológico e diminuição da saúde física (CARVALHO; PERSON, 2012; ARAÚJO et al., 2009; DY; ISENBERG, 2017; IDO; TADAKA, 2017).

Em pacientes com câncer terminal os familiares costumam ser os principais cuidadores, auxiliando o paciente com o autocuidado, a movimentação, transporte, comunicação, tarefas domésticas, compras, alimentação, financiamento, organização de compromissos e atividades sociais. Além dessas atividades comuns da rotina diária, os familiares também precisam auxiliar o paciente a lidar com os sintomas, medicamentos e cuidados médicos. Como consequência, os familiares sofrem elevada carga de trabalho, estresse e sofrem inúmeras dificuldades como questões físicas, sociais e financeiras (CARVALHO; PERSON, 2012; ARAÚJO et al., 2009; DY; ISENBERG, 2017; IDO; TADAKA, 2017).

Nesse contexto, é importante destacar o olhar para os familiares/cuidadores no processo de cuidados paliativos de pacientes terminais. Assim, questiona-se: (a) 
Quais as evidências apresentadas na literatura brasileira sobre a qualidade de vida de familiares/cuidadores de pacientes oncológicos que estão sob os Cuidados Paliativos? (b) Quais as formas de mensuração que estão sendo utilizadas para avaliar qualidade de vida dos familiares/cuidadores de pacientes oncológicos em Cuidados Paliativos?

A partir destas questões, definiu-se como objetivo deste estudo: identificar e analisar os instrumentos utilizados em estudos nacionais sobre a qualidade de vida dos familiares de pacientes oncológicos em Cuidados Paliativos.

\section{METODOLOGIA}

Realizou-se uma revisão integrativa da literatura (GRUPO ANIMA EDUCAÇÃO, 2014). A técnica de coleta de dados consistiu na pesquisa bibliográfica em bases de dados virtuais: Biblioteca Virtual de Saúde (BVS); US National Library of Medicine National Institutes of Health (PubMed/Medline) e Coordenação de Aperfeiçoamento de Pessoal de Nível Superior (CAPES).

A coleta dos dados foi realizada por meio de uma consulta no banco de dados bibliográficos BVS e PubMed/Medline com artigos indexados nas bases de dados Literatura Latino-Americana em Ciências de Saúde (LILACS), e Scientific Eletronic Library Online (SCIELO) e nos periódicos da CAPES. Os descritores empregados na busca dos artigos foram: cuidados paliativos; câncer; qualidade de vida; cuidador; família.

Os critérios de inclusão foram: (a) publicações nacionais diretamente relacionadas com o tema; (b) período de publicação: 2011 a 2018; (c) estudos com os temas sobre família/cuidadores (informais); (d) recursos de mensuração de qualidade de vida; (e) pacientes oncológicos acima de 18 anos.

Critérios de exclusão: (a) estudos que não abordem o tema "família" e/ou "cuidador informal"; (b) estudos que não abordem o tema "qualidade de vida" e sua avaliação/mensuração; (c) estudos sobre Cuidados Paliativos que não sejam sobre 
pacientes oncológicos; (d) estudos que caracterizem populações que não sejam brasileiras; (e) que não sejam dos últimos dez anos; (f) que sejam revisões de literatura; e ( $\mathrm{g}$ ) que se refiram a amostras de indivíduos menores de 18 anos.

Os estudos selecionados foram analisados de forma qualitativa, com base na interpretação das autoras.

A apresentação dos resultados foi elaborada de modo descritivo, em ordem cronológica de publicação, com base nos objetivos e resultados dos estudos selecionados.

\section{RESULTADOS}

Os resultados da busca, com a aplicação dos critérios de inclusão e exclusão, possibilitaram a seleção de 12 publicações para o presente estudo, como descrito no fluxograma abaixo.

Figura 1: Fluxograma (Flow chart) da busca nas bases de dados virtuais

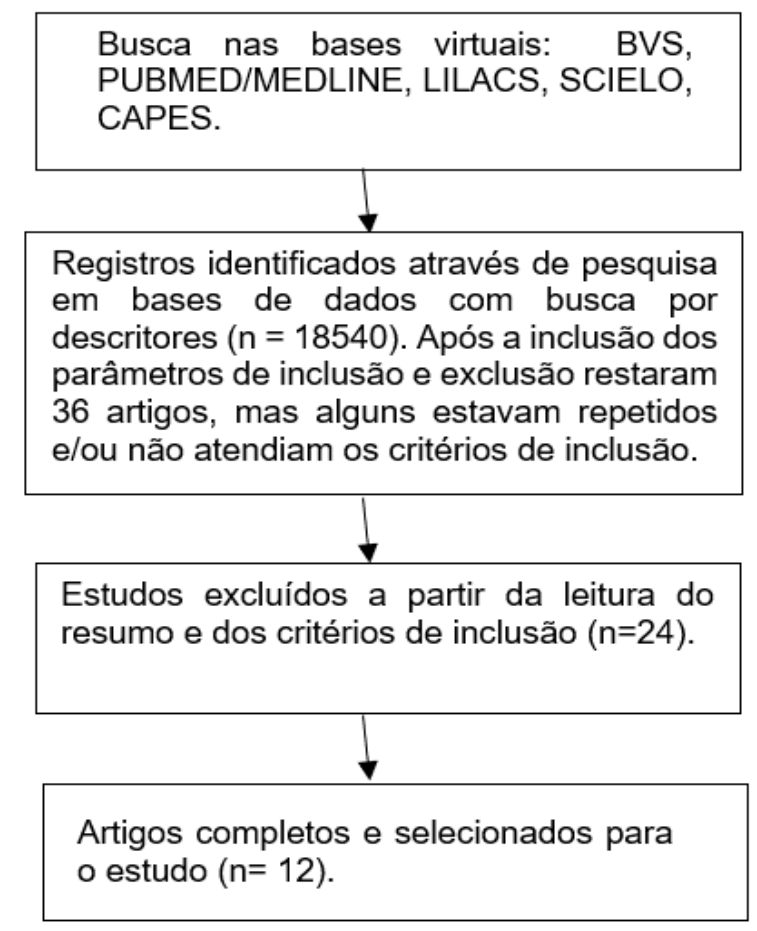

Fonte: elaboração das autoras. 
A tabela 1 apresenta uma descrição dos resultados da busca nas bases de dados virtuais.

Tabela 1: Resultados das buscas nas bases de dados virtuais

\begin{tabular}{|l|l|l|}
\hline BASE DE DADOS & ARTIGOS LISTADOS & ARTIGOS SELECIONADOS \\
\hline PUBMED/MEDLINE & 1017 & 0 \\
\hline LILACS & 444 & 1 \\
\hline SCIELO & 42 & 6 \\
\hline BVS & 37 & 2 \\
\hline CAPES & 17000 & 3 \\
\hline TOTAL & 18540 & 12 \\
\hline
\end{tabular}

Fonte: elaboração das autoras.

A busca inicial, sem os parâmetros de inclusão/exclusão descritos na metodologia, resultou em 18540 artigos indexados. Desse modo, foram aplicados os parâmetros de inclusão e exclusão, sendo que os parâmetros relacionados ao período de publicação (2011 a 2018) e de que deveriam ser publicações nacionais apenas, eliminaram a grande maioria dos estudos automaticamente, resultando em 36 artigos indexados.

A partir da seleção dos 36 artigos foi realizada uma leitura dos títulos e resumos dos mesmos e, desse modo, foram excluídos 24 artigos que não atendiam aos objetivos desta pesquisa, restando 12 artigos selecionados para análise.

A tabela 2 apresenta uma síntese dos 12 artigos selecionados para o presente estudo, considerando: autor/data, título, instrumentos utilizados e conclusões.

Tabela 2: Descrição dos estudos selecionados

\begin{tabular}{|c|c|c|c|}
\hline AUTOR/DATA & TÍTULO & $\begin{array}{l}\text { INSTRUMENTOS } \\
\text { UTILIZADOS }\end{array}$ & CONCLUSÕES \\
\hline 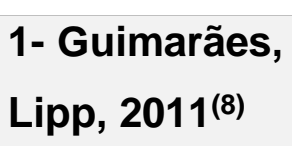 & $\begin{array}{l}\text { Um olhar sobre } 0 \\
\text { cuidador }\end{array}$ & $\begin{array}{l}\text { Entrevista } \\
\text { inventário }\end{array}$ & $\begin{array}{l}\mathrm{O} \text { ato de cuidar gera } \\
\text { mudanças que criam }\end{array}$ \\
\hline
\end{tabular}




\begin{tabular}{|c|c|c|c|}
\hline & $\begin{array}{l}\text { pacientes } \\
\text { oncológicos }\end{array}$ & $\begin{array}{lr}\text { sintomas } & \text { de } \\
\text { estresse } & \text { para } \\
\text { adultos de } & \text { Lipp } \\
\text { (ISSL) } & \end{array}$ & $\begin{array}{l}\text { dificuldades } \\
\text { necessidades } \\
\text { específicas dos } \\
\text { cuidadores. É preciso } \\
\text { que os profissionais } \\
\text { se preocupem } \\
\text { também com o bem- } \\
\text { estar e qualidade de } \\
\text { vida dos cuidadores. }\end{array}$ \\
\hline $\begin{array}{l}\text { 2- Magalhaes, } \\
\text { Franco, } \\
\text { 2012(9) }^{(91}\end{array}$ & $\begin{array}{l}\text { Experiência de } \\
\text { profissionais de } \\
\text { familiares } \\
\text { pacientes em } \\
\text { cuidados paliativos }\end{array}$ & $\begin{array}{l}\text { Entrevista e grupo } \\
\text { focal }\end{array}$ & $\begin{array}{l}\text { Os cuidadores } \\
\text { também necessitam } \\
\text { de acompanhamento } \\
\text { terapêutico, para que } \\
\text { as questões } \\
\text { relacionadas à } \\
\text { qualidade de vida } \\
\text { dessas pessoas } \\
\text { sejam refletidas. }\end{array}$ \\
\hline $\begin{array}{l}\text { 3- Sales et al., } \\
2012^{(10)}\end{array}$ & $\begin{array}{l}\text { Cuidado de } \\
\text { enfermagem } \\
\text { oncológico na ótica } \\
\text { do cuidador familiar } \\
\text { no contexto } \\
\text { hospitalar }\end{array}$ & Entrevista & $\begin{array}{l}\text { cuidado com } \\
\text { familiares, que atuam } \\
\text { como cuidadores de } \\
\text { pacientes terminais, } \\
\text { não se restringe } \\
\text { apenas às ações de } \\
\text { enfermagem, mas } \\
\text { também } \\
\text { medidas envolve } \\
\text { administrativas e de } \\
\text { infraestrutura } \\
\text { hospitalar para seu } \\
\text { bem-estar. }\end{array}$ \\
\hline
\end{tabular}




\begin{tabular}{|c|c|c|c|}
\hline $\begin{array}{l}\text { 4- Silva et al., } \\
2012^{(11)}\end{array}$ & $\begin{array}{l}\text { Análise do cuidado } \\
\text { de enfermagem e da } \\
\text { participação dos } \\
\text { familiares na atenção } \\
\text { paliativa oncológica }\end{array}$ & Entrevista & $\begin{array}{l}\text { O atendimento de } \\
\text { enfermagem aos } \\
\text { familiares do paciente } \\
\text { terminal deve iniciar } \\
\text { no diagnóstico } \\
\text { visando o bem-estar } \\
\text { desses } \\
\text { familiares/cuidadores. }\end{array}$ \\
\hline $\begin{array}{l}\text { 5- Ponte, } \\
\text { Ribeiro, } \\
\text { 2014(12) }\end{array}$ & $\begin{array}{l}\text { O bem-estar do } \\
\text { doente seguido em } \\
\text { cuidados paliativos } \\
(\mathrm{CP}) \text { : comparação } \\
\text { entre a perspectiva } \\
\text { do doente e da } \\
\text { família }\end{array}$ & $\begin{array}{l}\text { Entrevista e grupo } \\
\text { focal }\end{array}$ & $\begin{array}{l}\text { O cuidado paliativo } \\
\text { deve englobar } \\
\text { também a qualidade } \\
\text { de vida dos familiares } \\
\text { e não apenas do } \\
\text { paciente terminal. }\end{array}$ \\
\hline $\begin{array}{l}\text { 6- Marchi et } \\
\text { al., 2016(13) }\end{array}$ & $\begin{array}{l}\text { Significado de ser- } \\
\text { cuidador de familiar } \\
\text { com câncer e } \\
\text { dependente: } \\
\text { contribuições para a } \\
\text { paliação }\end{array}$ & $\begin{array}{l}\text { Entrevista } \\
\text { (fenomenológica) }\end{array}$ & $\begin{array}{l}\text { Os cuidadores zelam } \\
\text { pelo bem-estar do } \\
\text { paciente e sofrem } \\
\text { junto com o doente, } \\
\text { assim eles também } \\
\text { necessitam de } \\
\text { cuidados } \\
\text { especializados. }\end{array}$ \\
\hline $\begin{array}{l}\text { 7- Pinheiro et } \\
\text { al., } 2016^{(14)}\end{array}$ & $\begin{array}{l}\text { Paciente oncológico } \\
\text { em cuidados } \\
\text { paliativos: a } \\
\text { perspectiva do } \\
\text { familiar cuidador }\end{array}$ & Entrevista & $\begin{array}{l}\text { É necessário dar } \\
\text { apoio aos } \\
\text { cuidadores/familiares } \\
\text { para que eles } \\
\text { consigam enfrentar a } \\
\text { doença e o período de } \\
\text { luto. }\end{array}$ \\
\hline
\end{tabular}




\begin{tabular}{|c|c|c|c|}
\hline $\begin{array}{l}\text { 8- Borges et } \\
\text { al., } 2017^{(15)}\end{array}$ & $\begin{array}{l}\text { Sobrecarga do } \\
\text { cuidador familiar: a } \\
\text { sobrecarga de cuidar } \\
\text { de pacientes com } \\
\text { câncer de pulmão, de } \\
\text { acordo com o estágio } \\
\text { do câncer e a } \\
\text { qualidade de vida do } \\
\text { paciente }\end{array}$ & 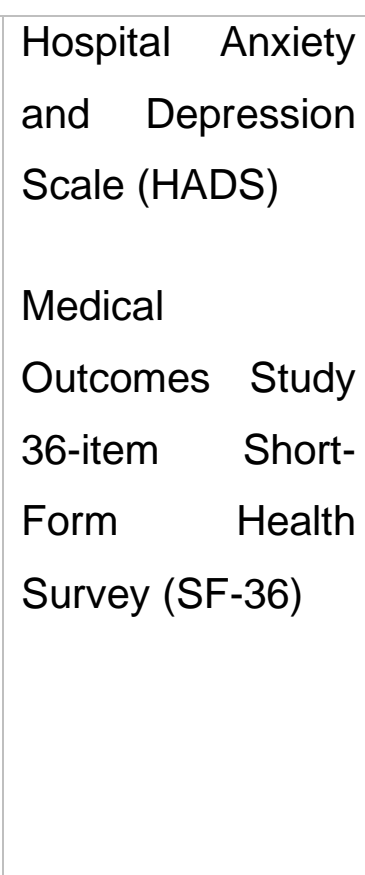 & $\begin{array}{lr}\text { Os } & \text { cuidadores } \\
\text { apresentaram níveis } & \text { elevados } \\
\text { ansiedade } & \text { de } \\
\text { depressão, } & \text { que } \\
\text { aumentaram } \\
\text { conforme o avanço da } \\
\text { doença do paciente, } \\
\text { comprometendo sua } \\
\text { qualidade de vida e } \\
\text { aumentando } \\
\text { estresse. }\end{array}$ \\
\hline $\begin{array}{l}\text { 9- Figueiredo } \\
\text { et al., } 2017^{(16)}\end{array}$ & $\begin{array}{l}\text { Como posso ajudar? } \\
\text { Sentimentos } \\
\text { experiências do } \\
\text { familiar cuidador de } \\
\text { pacientes } \\
\text { oncológicos }\end{array}$ & Entrevista & $\begin{array}{l}\text { O cuidado ao paciente } \\
\text { oncológico também } \\
\text { engloba os familiares } \\
\text { com apoio físico e } \\
\text { psicossocial, } \\
\text { proporcionando à } \\
\text { família qualidade de } \\
\text { vida durante o } \\
\text { tratamento. }\end{array}$ \\
\hline $\begin{array}{l}\text { 10- Furtado, } \\
\text { Leite, } 2017^{(17)}\end{array}$ & $\begin{array}{lr}\text { Cuidados } & \text { paliativos } \\
\text { sob a ótica de } & \text { de } \\
\text { familiares } & \text { de } \\
\text { pacientes } & \text { com } \\
\text { neoplasia de pulmão }\end{array}$ & Entrevista & $\begin{array}{l}\text { É preciso um olhar } \\
\text { mais atento também } \\
\text { para os familiares que } \\
\text { passam pelo } \\
\text { sofrimento juntamente } \\
\text { com o doente. }\end{array}$ \\
\hline $\begin{array}{l}\text { 11- Oliveira et } \\
\text { al., } 2017^{(18)}\end{array}$ & $\begin{array}{l}\text { Atendimento } \\
\text { domiciliar oncológico: } \\
\text { percepção de } \\
\text { familiares/cuidadores }\end{array}$ & $\begin{array}{l}\text { Entrevista } \\
\text { semiestruturada }\end{array}$ & $\begin{array}{l}\text { A rotina dos } \\
\text { cuidadores } \\
\text { demonstrou que sua } \\
\text { qualidade de vida foi }\end{array}$ \\
\hline
\end{tabular}




\begin{tabular}{|c|c|c|c|}
\hline & $\begin{array}{ll}\text { sobre } & \text { cuidados } \\
\text { paliativos } & \end{array}$ & & $\begin{array}{l}\text { prejudicada, pois } \\
\text { foram observadas } \\
\text { mudanças negativas } \\
\text { na vida pessoal dos } \\
\text { cuidadores/familiares, } \\
\text { tais como perda de } \\
\text { emprego } \\
\text { negligência com a } \\
\text { própria vida para } \\
\text { cuidar do paciente. }\end{array}$ \\
\hline $\begin{array}{l}\text { 12- Meneguin } \\
\text { et al., } 2018^{(19)}\end{array}$ & $\begin{array}{l}\text { Percepção de } \\
\text { pacientes } \\
\text { oncológicos em } \\
\text { cuidados paliativos } \\
\text { sobre qualidade de } \\
\text { vida }\end{array}$ & Entrevista & $\begin{array}{l}\text { A concepção de } \\
\text { qualidade de vida é } \\
\text { subjetiva, atrelada a } \\
\text { valores pessoais e } \\
\text { influenciada pelas } \\
\text { repercussões do } \\
\text { processo saúde- } \\
\text { doença. }\end{array}$ \\
\hline
\end{tabular}

Fonte: elaboração das autoras.

\section{DISCUSSÃO}

A prestação de cuidados paliativos ao paciente com câncer é complexa e requer preparo, especialmente de ordem emocional. Portanto, quando os familiares se tornam, inesperadamente, cuidadores, eles podem ter sua qualidade de vida prejudicada, tanto no seu bem-estar-físico quanto psicossocial (GUIMARÃES; LIPP, 2011; MENEGUIN; MATOS; FERREIRA, 2018).

Estudos acerca da população em questão revelaram que os familiares/cuidadores de pacientes com câncer terminal apresentam níveis mais elevados de depressão, ansiedade, disfunção do sono, pior estado de saúde, bem como menor qualidade de 
vida em comparação com os familiares não cuidadores (GUIMARÃES; LIPP, 2011; SALES et al., 2012; MARCHI et al., 2016).

Para mensurar a qualidade de vida dos familiares/cuidadores de pacientes oncológicos, a maioria dos estudos selecionados utilizou entrevista (GUIMARÃES; LIPP, 2011; PINHEIRO et al., 2016; FIGUEIREDO et al., 2016; MENEGUIN; MATOS; FERREIRA, 2018), um estudo conciliou a entrevista com o inventário de sintomas de estresse (GUIMARÃES; LIPP, 2011); um utilizou apenas as escalas: HADS e SF-36 (BORGES et al., 2017) para avaliação de fenômenos como ansiedade e depressão; dois estudos utilizaram a entrevista individual em conjunto com grupos focais (MAGALHAES; FRANCO, 2012; PONTE; RIBEIRO, 2014); mas, 6 artigos dos estudos empregou a entrevista semiestruturada (GUIMARÃES; LIPP, 2011; MAGALHAES; FRANCO, 2012; SILVA et al., 2012; PINHEIRO et al., 2016; FURTADO; LEITE, 2017; OLIVEIRA et al., 2017), outros 2 estudos (SALES et al., 2012; FIGUEIREDO et al., 2017) empregaram a entrevista aberta com apenas uma (FIGUEIREDO et al., 2017) ou duas (SALES et al., 2012) perguntas norteadoras ("Como está sendo para você conviver com seu familiar com o diagnóstico de câncer?" (FIGUEIREDO et al., 2017) "Como é para você a assistência de enfermagem oferecida dentro do ambiente hospitalar?" (SALES et al., 2012) e "Como você gostaria que fosse essa assistência no hospital?" (SALES et al., 2012); um estudo (MARCHI et al., 2016) utilizou um formulário estruturado; e outro estudo (BORGES et al., 2017) utilizou a entrevista fenomenológica.

Observa-se assim que há uma predominância de estudos qualitativos sobre o tema, sinalizando uma forma de compreensão desse fenômeno. Entende-se que o uso dessa ferramenta se dê pela expectativa e crença de que a qualidade de vida para essa população seja de difícil compreensão. Sendo assim, um instrumento aberto e individualizado como entrevistas permite alcançar um entendimento amplo e aprofundado da experiência e suas idiossincrasias. Sugere-se assim que existe uma lacuna na literatura nacional (MAGALHAES; FRANCO, 2012; PINHEIRO et al., 2016; FIGUEIREDO et al., 2017; MENEGUIN; MATOS; FERREIRA, 2017) sobre o uso de 
escalas consolidadas para avaliação da qualidade de vida de familiares que tornamse cuidadores (BORGES et al., 2017).

Os artigos selecionados (GUIMARÃES; LIPP, 2011; MENEGUIN; MATOS; FERREIRA, 2017) são unânimes em abordar que os familiares/cuidadores de pacientes em estado terminal lidam com uma série de emoções e situações de estresse, relacionadas ao estado do paciente e com a questão da morte iminente. Tais questões mostraram-se vinculadas à qualidade de vida dos cuidadores devido ao estresse e ansiedade decorrentes da responsabilidade de cuidar. Os familiares/cuidadores também sofrem problemas relacionados à sua própria condição de saúde que se deteriora durante o processo de cuidado com o paciente terminal, problemas financeiros e sociais, uma vez que passam a não ter tempo para suas próprias atividades sociais, laborais e financeiras.

Para conhecer-se tal realidade o instrumento utilizado com maior frequência foi a entrevista. Observa-se com isso uma prevalência de dados qualitativos, com foco no aprofundamento do conhecimento dessa experiência de cuidar do paciente terminal.

Embora o cuidado paliativo de um paciente terminal seja uma experiência significativa para os familiares/cuidadores, associada ao bem-estar do paciente, também está associada à uma deterioração da qualidade de vida dos cuidadores o que pode implicar em problemas psicológicos como estresse, ansiedade e depressão (GUIMARÃES; LIPP, 2011; SALES et al., 2012; MARCHI et al., 2016). Esses efeitos sobre a qualidade de vida dos cuidadores também afetam, em última instância, a qualidade do cuidado recebido pelos pacientes terminais (FURTADO; LEITE, 2017; MENEGUIN; MATOS; FERREIRA, 2018).

Os objetivos dos cuidados paliativos, para qualquer população de pacientes, incluem a gestão da dor e dos sintomas, o estabelecimento de um sistema de apoio ao paciente e à unidade familiar, além da otimização da qualidade de vida de ambos (GUIMARÃES; LIPP, 2011; MENEGUIN; MATOS; FERREIRA, 2018). Contudo, muitas vezes a qualidade de vida dos familiares/cuidadores é comprometida 
(MAGALHAES; FRANCO, 2012; BORGES et al., 2017; OLIVEIRA et al., 2017; MENEGUIN; MATOS; FERREIRA, 2018).

Assim como para a equipe, o processo de cuidados paliativos pode ser altamente estressante e oneroso para os familiares devido ao fato de terem de lidar com a questão da morte de um ente querido, comprometendo a qualidade de vida geral desses familiares/cuidadores, o que inclui o bem-estar físico, psicológico, social, financeiro e espiritual (GUIMARÃES; LIPP, 2011; SILVA et al., 2012; MARCHI et al., 2016; MENEGUIN; MATOS; FERREIRA, 2018). O diagnóstico e o tratamento de câncer representam uma ampla gama de desafios para o paciente e sua família, tanto do ponto de vista médico como psicossocial. Os familiares do paciente assumem, frequentemente, um papel de cuidador para ajudá-lo a suportar o fardo da doença tanto quanto possível (MAGALHAES; FRANCO, 2012; MENEGUIN; MATOS; FERREIRA, 2018).

A experiência do cuidado de um paciente terminal não depende apenas dos recursos e demandas de cuidado disponíveis, mas, também, da dinâmica familiar e da resiliência do cuidador isso atrelado à capacidade de resistir às crises e conseguir se adaptar, bem como de lidar com os problemas existentes. Esse é um processo difícil que demanda acompanhamento não apenas do paciente terminal e seus cuidadores (MAGALHAES; FRANCO, 2012; PINHEIRO et al., 2016).

Muitos familiares/cuidadores adotam o papel de cuidador com pouca ou nenhuma preparação, sem os conhecimentos adequados, sem recursos ou habilidades apropriadas e até sem motivação (MAGALHAES; FRANCO, 2012; MENEGUIN; MATOS; FERREIRA, 2018). Isso torna o processo de cuidados paliativos difícil para os familiares/cuidadores que, além de terem de lidar com a morte iminente do paciente, ainda se deparam com seus próprios conflitos emocionais e o estresse inerente da situação e do vínculo que possuem (BORGES et al., 2017; MENEGUIN; MATOS; FERREIRA, 2018).

Os estudos realizados por Guimarães et al. (2011); Magalhães et al. (2012); Silva et al. (2012); Ponte e Ribeiro (2014); Pinheiro et al. (2016) e Figueiredo et al. (2017) não 
descreveram as limitações de suas pesquisas. Outros estudos, como Borges et al. (2017) destacaram as limitações quando relacionadas ao desenho da pesquisa que não permitiu determinar a estabilidade do envolvimento dos cuidadores familiares ao longo do tempo. Outra limitação é que não foram considerados múltiplos cuidadores na análise. A limitação descrita por Furtado et al. (2017) sobre seu estudo estava relacionada ao fato de somente terem sido incluídos relatos de cuidadores de pacientes oncológicos próximos ao final da vida.

Os familiares/cuidadores, na tentativa de melhorar a qualidade de vida e garantir o bem-estar do paciente terminal, negligenciam sua própria vida, deixando de cuidar de sua saúde, relegam seus afazeres domésticos e atividades sociais (GUIMARAES; LIPP, 2011; PINHEIRO et al., 2016). Esse processo de esquecer de si mesmo para cuidar do outro gera muitas emoções e conflitos internos nos familiares, pois eles lidam com a perda de um ente querido e, concomitantemente, têm de lidar com uma série de outros problemas (físicos, mentais, sociais e financeiros) decorrentes desse processo de cuidar de um paciente terminal (GUIMARAES; LIPP, 2011; PINHEIRO et al., 2016).

Algumas limitações dos estudos analisados estavam relacionadas à falta de generalizações (SALES et al., 2012; MARCHI et al., 2016; OLIVEIRA et al., 2017). Nesse sentido, Marchi et al. (2016) afirmaram que sua pesquisa ficou restrita ao tempo e espaço das vivências dos cuidadores, impossibilitando a realização de generalizações do seu conteúdo com outras equipes da Estratégia Saúde da Família (ESF).

\section{CONSIDERAÇÕES FINAIS}

Com os resultados da revisão integrativa da literatura concluiu-se que:

- Os familiares/cuidadores de pacientes oncológicos em fase terminal, que recebem cuidados paliativos, têm sua qualidade de vida comprometida devido ao estresse físico, psicológico e econômico, aos quais são submetidos nesse 
período. Além disso, o fator mais estressante é ter de lidar com a questão da morte iminente do paciente.

- Como consequência, os familiares/cuidadores de pacientes que recebem cuidados paliativos também necessitam de atenção e cuidados como suporte emocional e até mesmo cuidados médicos, uma vez que podem ter sua saúde, física e psicológica, prejudicada durante o processo de acompanhamento de um paciente terminal.

- Os estudos foram na sua maioria qualitativos.

- No Brasil, a ferramenta mais utilizada para mensurar a qualidade de vida dos familiares/cuidadores de pacientes em cuidados paliativos é a entrevista, que pode ser acompanhada ou não de outros instrumentos de análise, como por exemplo: o grupo focal.

- A maioria das publicações utiliza predominantemente a entrevista como fonte de dados para avaliar a qualidade de vida dos familiares/cuidadores. A utilização de dados quantitativos poderia compor a análise do fenômeno de forma complementar.

Para trabalhos futuros, sugere-se a ampliação da revisão integrativa, incluindo estudos internacionais, para que seja possível comparar as ferramentas de mensuração utilizadas no Brasil com as utilizadas em outros países, bem como verificar a existência de distintos delineamentos acerca do tema. De tal forma, possibilitará a ampliação do conhecimento dos instrumentos de mensuração para a avaliação da qualidade de vida dos familiares/cuidadores e suas possibilidades e limitações.

\section{REFERÊNCIAS}

ARAUJO, LZS, ARAUJO, CZS, SOUTO, AKBA, OLIVEIRA, MS. Cuidador principal de paciente oncológico fora de possibilidade de cura, repercussões deste encargo. Revista Brasileira de Enfermagem, Brasília. 2009;62:32-7.

BORGES, EL, FRANCESCHINI, J, COSTA, LHD, FERNANDES, ALG, JAMNIK, S, SANTORO, IL. Sobrecarga do cuidador familiar: a sobrecarga de cuidar de pacientes 
com câncer de pulmão, de acordo com o estágio do câncer e a qualidade de vida do paciente. J Bras Pneumol. 2017;43(1):18-23.

CARVALHO, RT, PARSONS, HA. Manual de cuidados paliativos ANCP. $2^{\underline{a}}$ Ed. Ampliada e atualizada. Academia Nacional de Cuidados Paliativos, 2012.

DY, SM, ISENBERG, SR, AL, HAMAYEL, NA. Palliative Care for Cancer Survivors. Medical Clinics of North America. 2017;101(6): 1181-1196.

FIGUEIREDO, T, SILVA, AP, SILVA, RMR, SILVA, JJ, SILVA, CSO, ALCÂNTARA, DDF, SOUZA, LPS, SOUZA, AAM. Como posso ajudar? Sentimentos e experiências do familiar cuidador de pacientes oncológicos. ABCS Health Sci. 2017;42(1):34-39.

FURTADO, MEMF, LEITE, DMC. Cuidados paliativos sob a ótica de familiares de pacientes com neoplasia de pulmão. Interface comunicação saúde educação. 2017;21(63):969-980.

GRUPO ANIMA EDUCAÇÃO. Manual revisão bibliográfica sistemática integrativa: a pesquisa baseada em evidências. Belo Horizonte: Grupo Anima Educação, 2014.

GUIMARÃES, CA, LIPP, MEN. Um olhar sobre o cuidador de pacientes oncológicos recebendo cuidados paliativos. Psicologia: Teoria e Prática. 2011;13(2):50-62.

ITO, E, TADAKA, E. Quality of life among the family caregivers of patients with terminal cancer at home in Japan. Japan Journal of Nursing Science. 2017;14(4):341-352.

MAGALHAES, SB, FRANCO, ALS. Experiência de profissionais e familiares de pacientes em cuidados paliativos. Arquivos Brasileiros de Psicologia, Rio de Janeiro. 2012;64(3):94-109.

MARCHI, JA, PAULA, CC, PERLINI, NMOG, SALES, CA. Significado de ser-cuidador de familiar com câncer e dependente: contribuição para a paliação. Texto Contexto Enferm. 2016;25(1):e0760014. 
MENEGUIN, S, MATOS, TDS, FERREIRA, MLSM. Percepção de pacientes oncológicos em cuidados paliativos sobre qualidade de vida. Revista Brasileira de Enfermagem REBEn. 2018;71(4):2114-20.

OLIVEIRA, MBP, SOUZA, NR, BUSHATSKY, M, DÂMASO, BFR, BEZERRA, DM, BRITO, JA. Atendimento domiciliar oncológico: percepção de familiares/cuidadores sobre cuidados paliativos. Esc Anna Nery. 2017;21(2):e20170030.

PINHEIRO, MLA, MARTINS, FDP, RAFAEL, CMO, LIMA, UTS. Paciente oncológico em cuidados paliativos: a perspectiva do familiar cuidador. Revista de Enfermagem UFPE On Line, Recife. 2016;10(5):1749-5.

PONTE, ACSLC, RIBEIRO, JLP. O bem-estar do doente seguido em cuidados paliativos (CP): comparação entre a perspectiva do doente e da família. Psicologia, Saúde \& Doença. 2014;15(1):97-110.

RANALHO, L. Improving the Quality of End-of-Life Care in Pediatric Oncology Patients Through the Early Implementation of Palliative Care. Journal of Pediatric Oncology Nursing. 2017;34(6):374-380.

SALES, CA, GROSSI, ACM, ALMEIDA, CSL, SILVA, JDD, MARCON, SS. Cuidado de enfermagem oncológico na ótica do cuidador familiar no contexto hospitalar. Acta Paul Enferm. 2012;25(5):736-742.

SILVA, MM, MOREIRA, MC, LEITE, JL, ERDMANN, AL. Análise do cuidado de enfermagem e da participação dos familiares na atenção paliativa oncológica. Texto Contexto Enferm, Florianópolis. 2012;21(3):658-666.

WORLD HEALTH ORGANIZATION. GLOBOCAN 2012: Estimated Cancer Incidence, Mortality and Prevalence Worldwide 2012. France: WHO. Acesso em 04/12/2017. Disponível em: <http://globocan.iarc.fr/Pages/fact_sheets_cancer.aspx>.

Enviado: Setembro, 2020.

Aprovado: Novembro, 2020. 